\title{
On the formation of massive stellar clusters
}

\author{
G. Tenorio-Tagle ${ }^{1}$, J. Palouš², S. Silich ${ }^{3}$, G. A. Medina-Tanco ${ }^{4}$, and C. Muñoz-Tuñón ${ }^{5}$ \\ ${ }^{1}$ Instituto Nacional de Astrofísica Optica y Electrónica, AP 51, 72000 Puebla, México \\ e-mail: gtt@inaoep.mx \\ 2 Astronomical Institute, Academy of Sciences of the Czech Republic, Boční II 1401, 14131 Praha 4, Czech Republic \\ ${ }^{3}$ Instituto Nacional de Astrofísica Optica y Electrónica, AP 51, 72000 Puebla, México \\ e-mail: silich@inaoep.mx \\ ${ }^{4}$ Instituto Astronômico e Geofísico, USP, Av. Miguel Stéfano 4200, 04301-904 Sao Paulo, Brazil \\ e-mail: gustavo@iagusp.usp.br \\ 5 Instituto de Astrofísica de Canarias, 38200 La Laguna, Tenerife, Spain \\ e-mail: cmt@ll.iac.es
}

Received 7 April 2002 / Accepted 14 July 2003

\begin{abstract}
Here we model a star forming factory in which the continuous creation of stars results in a highly concentrated, massive (globular cluster-like) stellar system. We show that under very general conditions a large-scale gravitational instability in the ISM, which triggers the collapse of a massive cloud, leads with the aid of a spontaneous first generation of massive stars, to a standing, small-radius, cold and dense shell. Eventually, as more of the collapsing matter is processed and incorporated, the shell becomes gravitationally unstable and begins to fragment, allowing the formation of new stars, while keeping its location. This is due to a detailed balance established between the ram pressure from the collapsing cloud which, together with the gravitational force exerted on the shell by the forming cluster, acts against the mechanical energy deposited by the collection of new stars. We present a full analysis of feedback and show how the standing shell copes with the increasing mechanical energy generated by an increasing star-formation rate. The latter also leads to a rapidly growing number of ionizing photons, and we show that these manage to ionize only the inner skin of the standing star-forming shell. We analyze the mass spectrum of fragments that result from the continuous fragmentation of the standing shell and show that its shape is well approximated at the high mass end by a power law with slope -2.25 , very close to the value that fits the universal IMF. Furthermore, it presents a maximum near to one solar mass and a rapid change towards a much flatter slope for smaller fragments. The self-contamination resultant from the continuous generation of stars is shown to lead to a large metal spread in massive $\left(\sim 10^{6} M_{\odot}\right)$ clusters, while clusters with a mass similar to $10^{5} M_{\odot}$ or smaller, simply reflect the initial metalicity of the collapsing cloud. This is in good agreement with the data available for globular clusters in the Galaxy. Other observables such as the expected IR luminosity and the $\mathrm{H}_{\alpha}$ equivalent width caused by the forming clusters are also calculated.
\end{abstract}

Key words. stars: formation - superstar clusters - supernovae: general - ISM: bubbles - globular clusters: general galaxies: starburst

\section{Introduction}

There is a mode of star formation that leads to massive $\left(10^{4} M_{\odot}-\right.$ a few $\left.10^{6} M_{\odot}\right)$, densely concentrated collections of stars. These have been named young massive clusters, superstar clusters and for the more massive ones the term starburst has also been used. The clusters are believed to have evolved from an interstellar cloud mass distribution $N \propto M^{-2}$ (Elmegreen \& Efremov 1997) and thus young clusters present a similar power law (see also Zhang \& Fall 1999) independent of the environment, while old (globular) clusters have a mass distribution that falls off towards low masses (Harris \& Pudritz 1994), perhaps due to evaporation within a Hubble time (Elmegreen \& Efremov 1997). As pointed out by Ho (1997) young super-star clusters are overwhelmingly luminous

Send offprint requests to: J. Palouš, e-mail: palous@ig.cas.cz concentrations of stars that present a typical half-light radius of about $3 \mathrm{pc}$, and a mass that ranges from a few times $10^{4} \mathrm{M}_{\odot}$ to $10^{6} M_{\odot}$. The brightest ones have luminosities up to two orders of magnitude higher than R136 in 30 Doradus. Similar super-star cluster properties have been inferred from HST-STIS observations of AGN (Colina et al. 2002), and from radio continuum measurements of ultra-compact HII regions not visible in optical images, which indicates that they are the youngest, densest and most highly obscured star formation events ever found (Kobulnicky \& Johnson 1999; Johnson et al. 2001). The massive concentrations imply a high efficiency of star formation which even after long evolutionary times permits the tight configuration that characterizes them, despite the impact of photo-ionization, winds and supernovae, believed to efficiently disperse the gas left over from star formation. Thus the selfgravity that results from the high efficiency of star formation 
is what keeps the sources bound together. This high efficiency also brings us to believe that the formation of young clusters should be either a delayed or a very rapid event, to avoid negative feedback (Larsen \& Richtler 2000). The observational evidence now points to such massive units of star formation $\left(\sim 10^{4}-10^{6} M_{\odot}\right)$ present at the excitation centers of blue compact and starburst galaxies such as M82 (de Grijs et al. 2001; O'Connell et al. 1995), and NGC 253 (Watson et al. 1996) as well as in galaxies of different types (see also Larsen \& Richtler 2000; Larsen 1999). Very similar entities have also been found in interacting galaxies. Perhaps the best example of these is the Antennae with a collection of young star clusters with a median effective radius of $4 \mathrm{pc}$ and ages of about $7 \mathrm{Myr}$ (Whitmore et al. 1999). These galaxies also show other much larger entities (with an outer radius of $450 \mathrm{pc}$ ) which are not addressed in this paper. This star-forming activity in which masses similar to the total gas content found in galactic giant molecular clouds are turned into stars, all in a very small volume ( $\sim$ few $\mathrm{pc})$ implies the rapid accumulation of matter before star formation and negative feedback affect the collapsing cloud. Here we suggest that the feedback from continuously created massive stars $\left(M_{*} \geq 10 M_{\odot}\right)$ can sustain a fragmenting small radius standing shell, giving origin to concentrated massive stellar clusters, with a universal IMF.

Formation of a bound stellar cluster from the supershell expanding out of the gaseous cloud has been discussed by Brown et al. $(1991,1995)$. In their concept stars are formed when the supershell has swept out the entire cloud and expanded beyond its original boundary. However, the physical mechanism responsible for the supershell fragmentation and formation of the second generation of stars remains uncertain. Here we give a thorough discussion of the supershell gravitational stability and show that the standing shell that forms due to the balance between the ejected mass ram pressure and the central cluster gravitational pull may become gravitationally unstable allowing the continuous formation of the new generation of stars.

Section 2 describes the stellar factory, its self-regulation and the physics that lead to its closure, once a massive compact cluster has formed. Section 3 deals with the spectrum of fragments (sizes and numbers) expected from the factory model and compares this result with observed values of the IMF. Selfcontamination is analyzed in Sect. 4, where strong predictions on the metallicity of the resultant clusters are given. Section 5 deals with other observables such as the expected IR luminosity and the $\left(\mathrm{H}_{\alpha}\right)$ equivalent width of the resultant clusters. Finally, our conclusions are drawn in Sect. 6.

\section{The star-forming factory}

Our model invokes pressure-bounded, self-gravitating, isothermal clouds, which may become gravitationally unstable if sufficiently compressed (Ebert 1955; Bonner 1956). The gravitational instability allows a large cloud $\left(M_{\mathrm{c}} \sim 10^{4}-10^{6} M_{\odot}\right)$ to enter its isothermal $\left(T_{\mathrm{c}} \sim 100 \mathrm{~K}\right)$ collapse phase (Larson 1969; Bodenheimer \& Steigart 1968; Foster \& Chevalier 1993; Elmegreen et al. 2000), thereby developing a density and velocity structure with the following characteristics: 1) A central region of constant density (the plateau) where the velocity increases linearly from zero $\mathrm{km} \mathrm{s}^{-1}$ at the center, to a maximum value $\left(v_{\max }=3.3 c_{\mathrm{c}}\right.$ where $c_{\mathrm{c}} \sim 1 \mathrm{~km} \mathrm{~s}^{-1}$ is the sound speed of the collapsing cloud) at the boundary. 2) A region of increasing size and constant maximum velocity $\left(v_{\max }\right)$, where the density falls off as $R^{-2}$ (the skirt). As the collapse proceeds and the density in region 1 becomes larger, the knee region in the density distribution, that separates zones (1) and (2), moves closer to the center of the configuration with an increasing speed. As the density in the plateau region $\left(\rho_{\mathrm{p}}\right)$ increases, unstable fragments begin to form. These will first (as $\rho_{\mathrm{p}}$ grows larger than $10^{-20} \mathrm{~g} \mathrm{~cm}^{-3}$ ) have a Jeans mass similar to those of massive stars: $M_{\text {Jeans }}(\mathrm{g})=8.5 \times 10^{22}\left(\frac{T_{\mathrm{c}}}{\mu}\right)^{1.5} \rho_{\mathrm{p}}^{-0.5}$.

Although the above equation does not take into account turbulence and magnetic fields as in Mac Low \& Klessen (2003), one can, as a first approach, assume that a first stellar generation with a sufficient number of massive stars $\left(M_{*}=100-10 M_{\odot}\right)$ will form spontaneously in the central plateau region where the $3 \mathrm{D}$ converging flow may trigger their gravitational instability. The stellar fragments will detach from the flow and, on time-scales of the order of a few times $10^{5} \mathrm{yr}$, will enter the main sequence. From then onwards, through their winds and terminal supernova ( $\mathrm{SN}$ ) explosions, they will begin to have an important impact on the collapsing cloud. For this to happen however, massive stars ought to form in sufficient numbers as to jointly stop the infall at least in the most central regions of the plateau. Otherwise, individual stars, despite their mechanical energy input rate, will unavoidably be buried by the infalling cloud, delaying the impact of feedback until more massive stars form. We thus assume that the first generation of massive stars is able to regulate itself by displacing and storing the high-density matter left over from star formation into a cool expanding shell, thereby limiting the number of sources in the first stellar generation. One can show that, given the high densities $\left(n \geq 10^{4} \mathrm{~cm}^{-3}\right)$ attained both in the wind and in the plateau region and the size of the latter $(\sim 1 \mathrm{pc})$ a mass $\sim 10^{3} M_{\odot}$ is available for a first generation of stars (with masses $\geq 10 M_{\odot}$ ). The shell will be driven by the momentum injected by the central wind sources, and the ionization front will rapidly become trapped within the expanding layer (see Sect. 2.1). Thus the two most disruptive agents thought to interrupt (Hoyle 1953) or even stop (Cox 1983; Larson 1987; Franco et al. 1997) the formation of stars are kept well under control by the infalling cloud. The large densities also promote a rapid radiative cooling within the shell, allowing low temperatures $(\sim 10 \mathrm{~K})$. The expanding layer is at all times confronted with the increasing density and larger velocity of the matter in the unperturbed plateau region, and it soon becomes rampressure confined. That is, it will soon happen that the central wind ram-pressure $\left(\rho_{\mathrm{w}} v_{\mathrm{w}}^{2}\right)$ exactly balances the infalling cloud plateau ram-pressure. From then onwards the shell of swept-up matter is forced to recede towards the stars, given the increasing density and velocity of the undisturbed collapsing plateau region, causing eventually the burial of the first stars. A more interesting situation arises if the number of sources in the first stellar generation is such that the final position of the layer of shocked matter is close to the knee of the density distribution $\left(R_{\mathrm{k}}\right)$, where both the infalling gas density $\left(\rho_{\mathrm{k}}\right)$ and 
velocity $\left(v_{\max }\right)$ attain their maximum values. There, near $R_{\mathrm{k}}$, the wind ram-pressure $\left(\rho_{\mathrm{w}} v_{\mathrm{w}}^{2}\right)$ will exactly balance the infalling cloud ram-pressure $\left(\rho_{\mathrm{k}} v_{\max }^{2}\right)$ when the mechanical luminosity $\left(L_{\star}\right)$ of the first spontaneous stellar generation approaches the critical value

$L_{\text {crit }}=2 \pi R_{\mathrm{k}}^{2} \rho_{\mathrm{k}} v_{\mathrm{w}} v_{\text {max }}^{2}$.

Star formation will suddenly stop as all plateau matter left over from star formation is now locked in the standing shell. Once the shell acquires this standing location, it will be able to process the infalling cloud mass. In this way, the mechanical energy deposited by the first generation of massive stars favors the accumulation of infalling cloud mass in the standing shell, which becomes gravitationally unstable.

In our steady-state model everything happens at the same time. Gravitationally bound fragments continuously form in the unstable shell (at $R=R_{\mathrm{k}}$ ) and then, due to their negligible cross-section, freely fall towards the center of the configuration as they evolve into stars. The larger number of sources continuously enhances the mechanical luminosity of the forming cluster and with it the amount of mass returned as a wind into the shell $\left(\dot{M}_{\mathrm{w}}\right)$. At the same time, the continuous fragmentation of the shell and the infall of the resultant fragments acts as a source of mass in the most central region of the collapsing cloud, and this rapidly modifies the balance previously established between the wind and the infalling gas ram pressures. Indeed the ram pressure exerted by the wind sources, in order to keep the shell at its standing location, will now have to balance not only the infalling gas ram pressure but also the gravitational force exerted on the shell by the increasing mass of the central star cluster:

$4 \pi R_{\mathrm{k}}^{2} \rho_{\mathrm{w}} v_{\mathrm{w}}^{2}=4 \pi R_{\mathrm{k}}^{2} \rho_{\mathrm{k}} v_{\max }^{2}+\frac{G M_{\mathrm{sh}} M_{\mathrm{sc}}}{R_{\mathrm{k}}^{2}}$.

In the steady-state regime considered here, the central star cluster mass, $M_{\mathrm{sc}}$, and the shell mass, $M_{\mathrm{sh}}$, are $M_{\mathrm{sc}}=4 \pi R_{\mathrm{k}}^{2} \rho_{\mathrm{k}} v_{\max } t$ and $M_{\mathrm{sh}}=4 \pi R_{\mathrm{k}}^{2} \Sigma_{\mathrm{sh}}$, where $\Sigma_{\mathrm{sh}}$ is the shell surface density and $t$ is the evolutionary time. Comparing the first and the second terms on the right-hand side of Eq. (2) one can show that very soon, after $t \geq v_{\max } /\left(4 \pi G \Sigma_{\mathrm{sh}}\right) \approx 10^{4}-10^{5} \mathrm{yr}$, the infall ram pressure becomes negligible compared to the gravitational pull provided by the forming cluster. Thus the shell becomes gravitationally bound with its mechanical equilibrium simply given by the equation

$4 \pi R_{\mathrm{k}}^{2} \rho_{\mathrm{w}} v_{\mathrm{w}}^{2}=\frac{G M_{\mathrm{sh}} M_{\mathrm{sc}}}{R_{\mathrm{k}}^{2}}$.

One can show that despite the increasing effective gravity, the shell remains stable against Rayleigh-Taylor modes, because the density of the shocked wind is larger than that of the shocked infalling cloud. Also, nonlinear thin shell instabilities (see Vishniac 1994) and their induced pressure perturbations will be overcome by gravitational forces.

From the new equilibrium condition (Eq. (3)), one can find how the ejected mass density grows with time at the standing radius $r=R_{\mathrm{k}}$

$\rho_{\mathrm{w}}(t)=\frac{G M_{\mathrm{sh}} \rho_{\mathrm{k}} v_{\mathrm{max}}}{R_{\mathrm{k}}^{2} v_{\mathrm{w}}^{2}} t=\frac{4 \pi G \Sigma_{\mathrm{sh}} \rho_{\mathrm{k}} v_{\mathrm{max}}}{v_{\mathrm{w}}^{2}} t$, and thus determine the mechanical energy input rate, $L_{\mathrm{eq}}=$ $\frac{1}{2} \dot{M}_{\mathrm{w}}(t) v_{\mathrm{w}}^{2}$, required to support the shell in its equilibrium state:

$L_{\mathrm{eq}}=\frac{1}{2} \dot{M}_{\mathrm{w}}(t) v_{\mathrm{w}}^{2}=8 \pi^{2} G \Sigma_{\mathrm{sh}} \rho_{\mathrm{k}} R_{k}^{2} v_{\mathrm{w}} v_{\mathrm{max}} t$

i.e., to support the shell against the gravitational pull exerted by the forming central star cluster, the mechanical luminosity would have to grow linearly with time.

A second constraint on the mechanical luminosity arises from a consideration of the star formation rate (SFR). This is defined by the sum of the two sources of mass at the shell: the rate at which the collapsing cloud is processed by the shell $\left(\dot{M}_{\text {in }}\right)$, which is a constant, and that rate at which mass is ejected by the star cluster $\left(\dot{M}_{\mathrm{w}}\right)$, which increases linearly with time. Thus,

$\operatorname{SFR}(t)=\dot{M}_{\mathrm{in}}+\dot{M}_{\mathrm{w}}=4 \pi R_{\mathrm{k}}^{2} \rho_{\mathrm{k}} v_{\max }\left(1+\frac{4 \pi G \Sigma_{\mathrm{sh}} t}{v_{\mathrm{w}}}\right)$,

is a function that increases also linearly with time. Such star formation rate defines the energy deposition $\left(L_{\mathrm{sc}}\right)$, expected from the growing central star cluster. Comparing $L_{\mathrm{eq}}$ (Eq. (5)) with $L_{\mathrm{sc}}$, one can then find the value of the shell surface density $\Sigma_{\mathrm{sh}} . L_{\mathrm{sc}}$ is to be derived from starburst synthesis models (Leitherer \& Heckman 1995; Mas-Hesse \& Kunth 1991; Silich et al. 2002) taking into consideration the SFR prescribed by (Eq. (6)) and an assumed metallicity of the ISM of the host galaxy.

Figure 1 shows that the energy input rate derived independently from the starburst synthesis models is in reasonable agreement with the equilibrium value (Eq. (5)) over a considerable span of time, particularly when $\Sigma_{\mathrm{sh}} \approx 0.7-1.1 \mathrm{~g} \mathrm{~cm}^{-2}$. In such cases both mechanical energy input rates agree to within less than a factor of two-three over almost 25-30 Myr.

A star-forming factory then results from a profound selfregulation that accounts for the mass continuously added to the forming cluster, as well as for the mechanical energy that results from this further addition of mass and its transformation into stars. Self-regulation keeps the shell at its standing location and thus with the same fragmenting properties, while the forming cluster remains hidden behind the shell and the collapsing cloud.

\subsection{The negative feedback caused by photoionization}

The increasing star formation rate also leads to a rapidly growing number of ionizing photons $\left(N_{\mathrm{sc}}\right)$. This has also been calculated (see Fig. 2a) using the starburst synthesis model (Silich et al. 2002) under the assumption of a linearly increasing star formation rate, as prescribed by relation (6). Clearly, the ionizing radiation may upset the shell fragmenting properties by simply changing, through photoionization, the temperature of the swept-up gas. Following Comeron (1997) and Tenorio-Tagle et al. (1999) we have derived the impact that such an increasing ionizing photon flux has on the collapsing shell. 


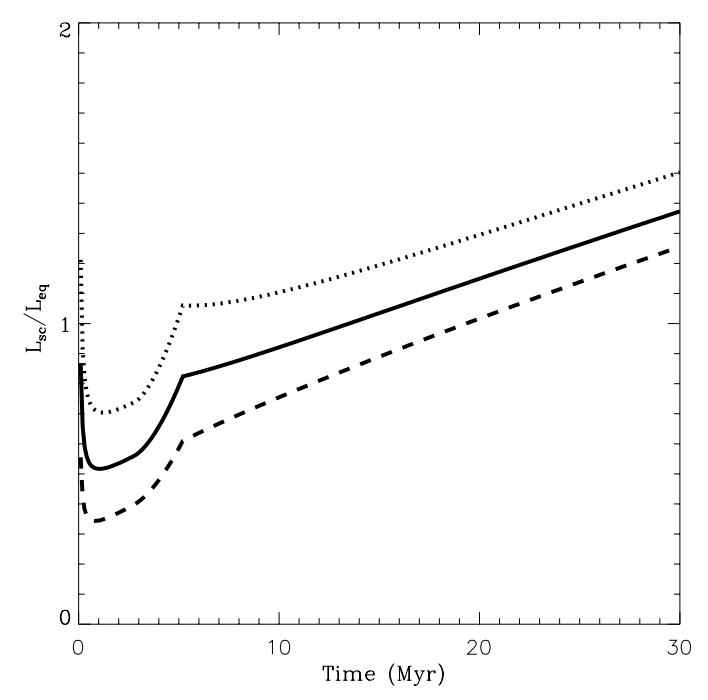

Fig. 1. Mechanical energy requirements. The figure shows the ratio $L_{\mathrm{sc}}$ (Eq. (6)) over $L_{\mathrm{eq}}$ (Eq. (5)). The $L_{\mathrm{sc}}$ values result from starburst synthesis models that assume a $S F R(t)$ as given by Eq. (6), for different values of $\Sigma_{\mathrm{sh}}=0.5$ (dotted lines), 0.7 (solid lines) and $1.1 \mathrm{~g} \mathrm{~cm}^{-2}$ (dashed lines). All models also assume upper and lower mass limits equal to $100 M_{\odot}$ and $1 M_{\odot}$ and a slope of -2.25 for the high mass end (as derived in Sect. 3). $L_{\mathrm{sc}}$ assumes mechanical energy input rate from a continuous star formation rate (Leitherer \& Heckman 1995) assuming a galaxy metallicity value $=0.25 Z_{\odot}$.

The shell density has been calculated from the momentum balance:

$\rho_{\mathrm{sh}}=\rho_{\mathrm{w}}\left(R_{k}\right)\left(\frac{v_{\mathrm{w}}}{c_{\mathrm{sh}}}\right)^{2}$.

where $\rho_{\mathrm{w}}$ as given by Eq. (4), is a function of time, and $c_{\mathrm{sh}}$ is the sound speed of the shell. The full thickness of the shell is

$l_{\mathrm{sh}}=\frac{\Sigma_{\mathrm{sh}}}{\mu_{\mathrm{n}} n_{\mathrm{sh}}}$,

where $\mu_{\mathrm{n}}$ and $n_{\mathrm{sh}}$ are the mean mass per particle and the shell number density, respectively. Thus the number of photons required for a complete ionization of the shell is:

$N_{\text {crit }}=\frac{4 \pi}{3}\left[\left(R_{\mathrm{k}}+l_{\mathrm{sh}}\right)^{3}-R_{\mathrm{k}}^{3}\right] n_{\mathrm{sh}}^{2} \alpha_{\beta}$,

where $\alpha_{\beta}=2.59 \times 10^{-13} \mathrm{~cm}^{3} \mathrm{~s}^{-1}$ is the recombination coefficient to all levels but the ground state (Osterbrock 1989). A comparison of the critical number of photons required for a complete ionization of the shell, with that of ionizing photons $\left(N_{\text {sc }}\right)$ emitted by the growing central star cluster $\left(N_{\text {sc }} / N_{\text {crit }}\right)$ as function of evolutionary time $(t)$ is shown in Fig. $2 b$. Throughout the evolution, this always remains $\ll 1$. This implies that the ionization front, despite the increasing number of photons, is trapped within the shell structure and furthermore, is only able to photo-ionize a narrow inner section, the inner skin, of the gravitationally unstable shell. It is worth noticing that a fraction of the UV flux will be absorbed within a free wind region, reducing further the number of the UV photons reaching the shell per unit area and per unit time. Therefore the two possible negative feedback mechanisms, the ionizing radiation and the mechanical energy of the forming cluster, remain at all times under control by the star-forming factory.
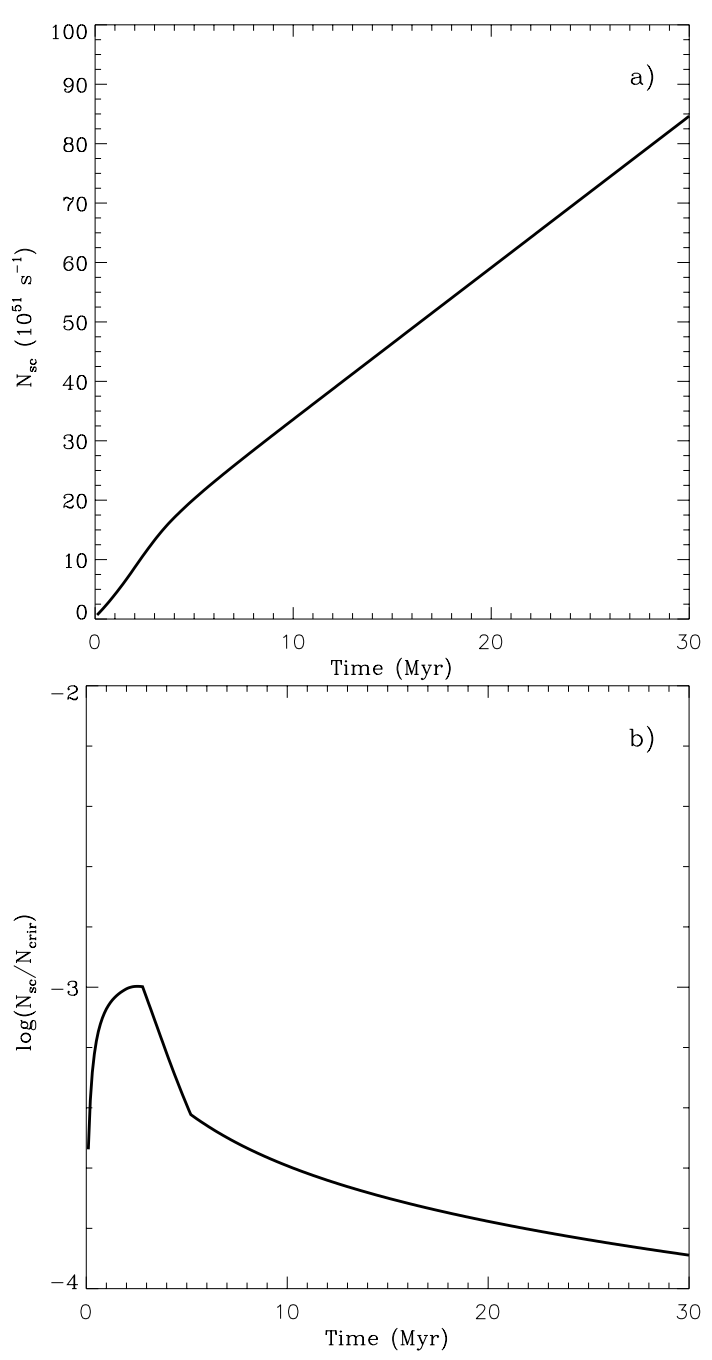

Fig. 2. The effects of photoionization. a) The ionizing radiation produced by the forming cluster $\left(N_{\mathrm{sc}}\right)$ as a function of time as derived from the starburst synthesis models under the same assumptions as those used in Fig. 1 and a $\left.\Sigma_{\mathrm{sh}}=0.7 \mathrm{~g} \mathrm{~cm}^{-2} ; \mathbf{b}\right)$ a comparison of the ratio of the photon flux $\left(N_{\mathrm{sc}}\right)$ and the number required to fully ionized the star forming shell $\left(N_{\text {crit }}\right)$, as a function of time.

\subsection{The size of the resultant clusters}

The factory stops operating either because small clouds $\left(M_{\mathrm{c}} \leq\right.$ $10^{5} M_{\odot}$ ) are rapidly processed by the standing shell or, in the case of larger clouds, because the mechanical energy input rate implied by the SFR condition $\left(L_{\mathrm{sc}}\right)$, here derived using starburst synthesis models, begins to largely exceed the luminosity required $\left(L_{\mathrm{eq}}\right)$ to keep the shell in its standing location. This latter possibility arises after $25 \mathrm{Myr}$ of evolution (see Fig. 1), when the luminosity generated by the increasing SFR begins to dominate the equilibrium condition, although not by more than a factor of 2. After this time the shell will lose its standing location and be disrupted as it accelerates into the skirt of the remaining cloud. Thus, the size of the largest resultant clusters in our factory model, is restricted to a few $10^{6} M_{\odot}$, the amount of cloud mass that can be processed by the standing shell within this time interval. 
Consequently, the half-light radius of the resultant cluster corresponds to the radius of the standing shell, and to the fraction of mass of the remaining cloud which is accelerated and removed from the original cloud when the mechanical energy input rate $L_{\mathrm{sc}}$ surpasses the equilibrium condition. Assuming that $1 / 2$ of the original cluster mass has been removed, the radius of the cluster doubles, reaching the value of a few pc. The final size of the cluster depends on its subsequent internal evolution and on the environment in the home galaxy.

Let us assume a cloud of $10^{6} M_{\odot}$ that has developed the plateau-knee-skirt structure during its isothermal $\left(T_{\mathrm{c}}=100 \mathrm{~K}\right)$ collapse phase. By the time the knee reaches a radius of $2 \mathrm{pc}$, $\rho_{\mathrm{p}}$ would be $\sim 10^{-20} \mathrm{~g} \mathrm{~cm}^{-3}$, and massive stars $\left(M_{*} \leq 100 M_{\odot}\right)$ will begin to appear at the center of the collapsing configuration. These will store the surrounding gas into an expanding shell, limiting the number of sources in the first stellar generation. The expanding shell becomes ram-pressure confined within the plateau. However, given the increasing density in the unperturbed plateau region, the shell must recede until it finds the knee of the density distribution, where both density and velocity of the incoming gas remain at a constant value. Here we assume a final shell standing at a distance of $1 \mathrm{pc}$. The density $\rho_{\mathrm{k}}$ would then be $\approx 6.68 \times 10^{-20} \mathrm{~g} \mathrm{~cm}^{-3}$, and the infalling velocity equals $3.9 \mathrm{~km} \mathrm{~s}^{-1}$. At this time the outer radius of the $10^{6} M_{\odot}$ cloud is $R_{\max } \approx M_{\mathrm{c}} /\left(4 \pi \rho_{\mathrm{k}} R_{\mathrm{k}}^{2}\right) \approx 90 \mathrm{pc}$. Assuming $v_{\mathrm{w}}=10^{8} \mathrm{~cm} \mathrm{~s}^{-1}$, the mechanical luminosity derived from Eq. (1) is $L \approx 5.8 \times 10^{37} \mathrm{erg} \mathrm{s}^{-1}$.

In the steady-state regime, the infalling mass ends up being transformed into stars at the rate at which it is processed by the standing shell $\dot{M}_{\mathrm{in}}=4 \pi R_{\mathrm{k}}^{2} \rho_{\mathrm{k}} v_{\max } \approx 4.4 \times$ $10^{-2} M_{\odot} \mathrm{yr}^{-1}$. An originally $10^{6} M_{\odot}$ cloud would then be processed in about $24 \mathrm{Myr}$, while the mechanical luminosity of the forming cluster increases almost linearly with time up to $6.3 \times 10^{40} \mathrm{erg} \mathrm{s}^{-1}$.

Given the self-similar solution of the isothermal collapse phase, all collapsing clouds are processed in a very similar manner, all with a similar IMF, and all of them causing a similar SFR rapidly increasing with time. Thus the only limitation on the mass of the resultant clusters is set by the time during which they are able to enhance their mechanical luminosity with time, to keep the fragmenting shell at its standing location. Note also that for a given plateau density, collapsing clouds present the same size plateau, regardless of the mass of the collapsing cloud $\left(M_{\mathrm{c}}\right)$. Thus, as the radius of the starforming shell is independent of the collapsing cloud mass, the mass-radius relation observed for GMCs is not reflected in the stellar clusters, as pointed out by Ashman \& Zepf (2001).

\section{The mass spectrum of fragments}

Mass accumulation leads to the gravitational instability of the standing shell with a well-defined mass and number of resultant fragments. The dispersion relation for gravitational instability of an expanding shell of radius $R$ is (Elmegreen 1994; Wünsch \& Palouš 2001): $\omega=-\frac{3 \dot{R}}{R}+\left(\frac{\dot{R}^{2}}{R^{2}}-\frac{\eta^{2} c_{\mathrm{sh}}^{2}}{R^{2}}+\frac{2 \pi G \Sigma_{\mathrm{sh}} \eta}{R}\right)^{1 / 2}$, where $\Sigma_{\mathrm{sh}}$ is the unperturbed surface density of the shell, $c_{\mathrm{sh}}$ its sound speed and $G$ is the gravitational constant. The condition for

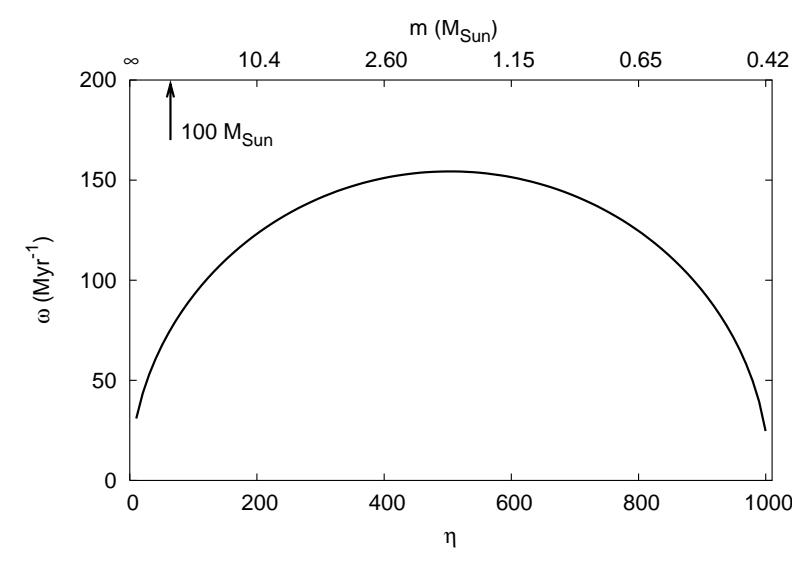

Fig. 3. The dispersion relation $\omega(\eta)$ for $c_{\text {sh }}$ corresponding to $T_{\mathrm{sh}}=$ $10 \mathrm{~K}, R_{\mathrm{k}}=1 \mathrm{pc}$ and $\Sigma_{\mathrm{sh}}=0.7 \mathrm{~g} \mathrm{~cm}^{-2} \cdot \eta_{\max }=503$, corresponds to the wavelength $\lambda=0.01 \mathrm{pc}$, gravitationally unstable mass $m=$ $\pi \Sigma_{\text {sh }} \lambda^{2}=1.6 M_{\odot}$, and the e-folding time of the growth of perturbations of $5 \times 10^{3} \mathrm{yr}$. The largest and smallest masses form at $\eta=62$ and 1005 corresponding to the mass range $0.39-150 M_{\odot}$. The upper axis indicates the (non-linear) mass scale, and the arrow the position of a $100 M_{\odot}$ fragment.

instability demands $\omega$ to be real and positive. The wavenumber $\eta$ is related to the perturbation wavelength $\lambda$ by $\eta=2 \pi R \lambda^{-1}$, and the e-folding time of the perturbation growth is $\omega^{-1}$. For a standing shell configuration (with $\dot{R}=0$ ) this reduces to

$\omega^{2}=-\frac{\eta^{2} c_{\mathrm{sh}}^{2}}{R^{2}}+\frac{2 \pi G \Sigma_{\mathrm{sh}} \eta}{R}$

The dispersion relation $\omega(\eta)$ is shown in Fig. 3. The fastest growing mode occurs for $\eta_{\max }=\frac{\pi G \Sigma_{\mathrm{s}} R}{c_{\mathrm{sh}}^{2}}$, corresponding to $\omega_{\max }=\frac{\pi G \Sigma_{\mathrm{sh}}}{c_{\mathrm{sh}}}$. Fragments of mass

$m=\pi \Sigma_{\mathrm{sh}} \lambda^{2}=4 \pi^{3} R^{2} \Sigma_{\mathrm{sh}} \eta^{-2}$

form with a frequency proportional to the growth rate, which is given by $\omega$, and is proportional to the shell surface available to accommodate fragments of a given wavelength, $\frac{R^{2}}{\lambda^{2}}=\frac{\eta^{2}}{4 \pi^{2}}$. Consequently the number of fragments $\Delta N$ formed per unit time, out of the standing shell, with the wavenumber from the interval $(\eta, \eta+\Delta \eta)$ is

$$
\begin{aligned}
\Delta N & =\left(\omega \frac{2 \eta}{4 \pi^{2}}+\frac{\eta^{2}}{4 \pi^{2}} \frac{\mathrm{d} \omega}{\mathrm{d} \eta}\right) \Delta \eta \\
& =\frac{\eta}{4 \pi^{2}}\left(2 \omega+\eta \frac{\mathrm{d} \omega}{\mathrm{d} \eta}\right) \Delta \eta
\end{aligned}
$$

The $\Delta \eta$ interval, can be expressed in terms of the corresponding $\Delta m$ by means of Eq. (11). And thus, Eqs. (11) and (12) imply the mass spectrum of fragments, or define the initial mass function $\xi(m)=\Delta N / \Delta m$.

$$
\begin{aligned}
\xi(m)= & Q \pi^{9 / 4} \Sigma_{\mathrm{sh}}^{3 / 2} R^{2} m^{-9 / 4} \\
& \times \frac{-3 \pi^{1 / 2} c_{\mathrm{sh}}^{2} m^{-1 / 2}+2.5 G \Sigma_{\mathrm{sh}}^{1 / 2}}{\left(-\pi^{1 / 2} c_{\mathrm{sh}}^{2} m^{-1 / 2}+G \Sigma_{\mathrm{sh}}^{1 / 2}\right)^{1 / 2}},
\end{aligned}
$$

where $Q$ is the normalization factor fixed by the star formation rate $(S F R)$. Note that a negative $\Delta m$ should be used for positive $\Delta \eta$. Thus, mass accumulation leads to the gravitational 


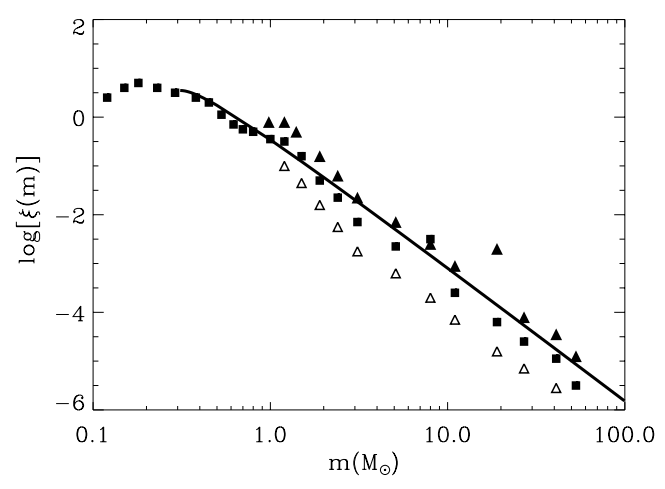

Fig. 4. The IMF $-\xi(m)-$ as given by Binney \& Merrifield (1998), is compared with our results from Eq. (13) normalized to the total cluster mass (solid line). The comparison assumes a $10^{6} M_{\odot}$ cloud, used below as an example, fully processed into stars during a time span of $24 \mathrm{Myr}$ (see text).

instability of the standing shell with a well defined mass and number of resultant fragments. From the dispersion relation of the linearized analysis of the hydrodynamical equations on the surface of the standing shell, the mass spectrum of gravitationally bound fragments presents a slope equal to -2.25 for massive objects. The distribution flattens in the neighborhood of $\eta_{\max }$ and peaks at $m=\frac{\pi c_{\mathrm{sh}}^{4}}{4 G^{2} \Sigma_{\mathrm{sh}}}$. The minimum mass, obtained from the condition that $\omega$ is positive, lies at $2 \eta_{\max }$. These results are in good agreement with the stellar mass distribution (see Fig. 4) inferred for star clusters (Moffat 1997; Hunter et al. 1997; Wyse 1997; Piotto \& Zoccali 1999; Paresce \& De Marchi 2000) and for the solar neighborhood (Salpeter 1955; Scalo 1986; Binney \& Merrifield 1998; Kroupa 2001, 2002).

\section{Self-contamination}

After $\sim 3$ Myr of evolution, the mass ejected from the star cluster $\left(\dot{M}_{\mathrm{w}}\right)$ becomes rapidly contaminated by the supernova explosions. We assume that on reaching the shell the metalenriched matter is immediately mixed with the gas coming from the collapsing cloud $\left(\dot{M}_{\text {in }}\right)$ which, depending on the host galaxy, may present a low metallicity. This leads to a continuously increasing abundance for every new generation of stars resulting from the fragmenting shell.

We assume that the gas ejected by stellar winds and $\mathrm{SNe}$ includes all the newly synthesized metals as well as metals distributed throughout the stellar hydrogen envelopes of the progenitor. The total mass ejection rate $\dot{M}_{\mathrm{w}}$ and the rate of metal ejection $\dot{M}_{\text {met }}$ for a central star cluster with a power-law initial mass function then are

$$
\begin{aligned}
\dot{M}_{\mathrm{w}}(t) & =\sum_{i} \frac{M_{i}}{\Delta t} \frac{M(t+\Delta t)^{2-\alpha}-M(t)^{2-\alpha}}{M_{\mathrm{low}}^{2-\alpha}-M_{\mathrm{up}}^{2-\alpha}}, \\
\dot{M}_{\mathrm{met}}= & \frac{(\alpha-2)}{M_{\mathrm{low}}^{2-\alpha}-M_{\mathrm{up}}^{2-\alpha}} \\
& \times \sum_{i} \frac{M_{i}}{\Delta t} \int_{M(t+\Delta t)}^{M(t)} Y_{\mathrm{met}}(m) m^{-\alpha} \mathrm{d} m,
\end{aligned}
$$

where $M_{i}$ is the mass of every new generation of stars, $t$ is the evolutionary time, $\Delta t$ is the time step, $M_{\text {low }}$ and $M_{\text {up }}$ are the lower and upper cut-off masses, respectively, $M(t)$ is the mass of the stars exploding after an evolutionary time $t$ (see Silich et al. 2002).

The evolution of the iron abundance of the forming star cluster (which can be compared with the available metallicities of galactic super-star clusters) can be approximated by means of an iron yield $Y_{\mathrm{Fe}}$. Here we use the Thielemann et al. (1992) model and extrapolate their results as constant yields for low $\left(<13 M_{\odot}\right)$ and high (>25 $M_{\odot}$ ) mass stars (see for details Silich et al. 2001).

We then adopt a helium normal abundance (one helium atom for every ten hydrogen atoms) and calculate the number of hydrogen and iron atoms mixing inside a shell at every time step

$\dot{N}_{\mathrm{H}}=\frac{\dot{M}_{\mathrm{tot}}}{1.1 \mu_{\mathrm{n}}}$,

$\dot{N}_{\mathrm{Fe}}=\frac{\dot{M}_{\mathrm{Fe}}}{A_{\mathrm{Fe}} m_{\mathrm{H}}}$,

where $\mu_{\mathrm{n}}=\frac{14}{11} m_{\mathrm{H}}$ is the mean mass per particle, $A_{\mathrm{Fe}}=55.4$ is the atomic number of iron in hydrogen mass units, and $m_{\mathrm{H}}$ is the hydrogen mass. The total mass input rate $\dot{M}_{\text {tot }}$, as well as the iron mass input rate $\dot{M}_{\mathrm{Fe}}$, includes both the matter ejected as a function of time by the star cluster and the mass coming from the collapsing cloud:

$\dot{M}_{\mathrm{tot}}(t)=\dot{M}_{\mathrm{w}}+\dot{M}_{\mathrm{in}}$,

$\dot{M}_{\mathrm{Fe}}(t)=\dot{M}_{\mathrm{Fe}, \mathrm{w}}+\dot{M}_{\mathrm{Fe}, \text { in }}$.

The iron abundance of every new generation of stars is

$\left[\frac{\mathrm{Fe}}{\mathrm{H}}\right]=\log \left(\frac{\dot{N}_{\mathrm{Fe}}}{\dot{N}_{\mathrm{H}}}\right)-\log \left(\frac{N_{\mathrm{Fe}}}{N_{\mathrm{H}}}\right)_{\odot}$,

where the Solar iron abundance is $\log \left(\frac{N_{\mathrm{Fe}}}{N_{\mathrm{H}}}\right)_{\odot}+12=7.448$ (Holweger 2001). Figure 5 presents the calculated iron abundance of the fragmenting shell, as a function of time, for galaxies with different ISM metallicities. As the clusters form from the ISM gas as that is contaminated by the supernova products from former stellar generations, the resultant metal spread in a cluster depends strongly on its final mass (upper axis in Fig. 5). In this way, clusters with a final mass smaller than $2.7 \times$ $10^{5} M_{\odot}$, processed within $3 \mathrm{Myr}$, will display the metal abundance that would reflect that of the ISM at the moment of formation. More massive clusters however, as their formation time may exceed the supernova era ( $\sim 3 \mathrm{Myr})$ from former stellar generations, will display a large metal spread in their sources. A cluster of $10^{6} M_{\odot}$ (indicated by an arrow in Fig. 5), requires $24 \mathrm{Myr}$ to complete its formation. If such a cluster forms in a low metallicity $\left(Z_{\mathrm{ISM}}=0.01 Z_{\odot}\right)$ galaxy for example, it will present stars with different $[\mathrm{Fe} / \mathrm{H}]$ abundance within the range $-2 \leq[\mathrm{Fe} / \mathrm{H}] \leq-0.13$, while equally massive clusters forming out of a more metal-rich ISM will show a correspondingly smaller relative metallicity spread (see Fig. 5). Note however that the spread caused during formation of the clusters depends strongly on the assumed metal yields. 


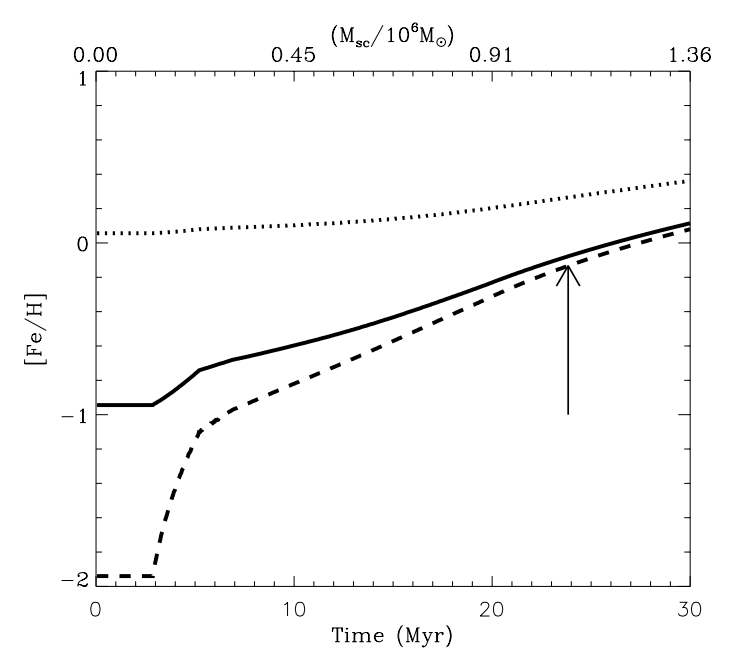

Fig. 5. The $[\mathrm{Fe} / \mathrm{H}]$ metallicity of the resultant clusters. The star forming shell metallicity as function of time for galaxies with different initial metal abundance: $Z_{\mathrm{ISM}}=0.01 Z_{\odot}$ (dashed line) $Z_{\mathrm{ISM}}=0.1 Z_{\odot}$ (Solid line) and $Z_{\mathrm{ISM}}=Z_{\odot}$ (dotted line). The resultant clusters, depending on their mass (upper axis), will show a metal spread that would strongly depend on the ISM original metallicity.

\section{Further observational properties}

Since the star-forming shell is dense, let us assume that there is enough dust to obscure the optical emission of the forming star cluster and transform it into the IR. The expected IR nebular luminosity would then result from the stellar ionizing flux and from the degraded cluster mechanical luminosity, thermalized at the reverse shock and then radiated away via effective gas cooling. Therefore the luminosity of the IR nebula associated for example to a $\sim 10^{6} M_{\odot}$ cluster may reach $\sim 10^{8} L_{\odot}$.

After formation (see Sect. 2.2) the newly formed star cluster will become visible in the optical line emission region. At such a time, the $\mathrm{H}_{\alpha}$ luminosities of a $10^{5} M_{\odot}$ and $10^{6} M_{\odot}$ star clusters would be $L_{\mathrm{H} \alpha} \approx 1.4 \times 10^{40} \mathrm{erg} \mathrm{s}^{-1}$ and $L_{\mathrm{H} \alpha} \approx$ $9.4 \times 10^{40} \mathrm{erg} \mathrm{s}^{-1}$, respectively, if all ionizing photons are trapped within the surrounding gas. The Lyman continuum rate of the $10^{6} M_{\odot}$ cluster is around $6.9 \times 10^{52} \mathrm{~s}^{-1}$, comparable to the Lyman continuum rate $\left(3 \times 10^{52} \mathrm{~s}^{-1}\right)$ derived from the radio emission flux of super-nebula NGC 5253 (Gorjian et al. 2001).

The continuous creation of stars in the star-forming factory model also leaves a foot-print on its $\mathrm{H}_{\alpha}$ equivalent width. Figure 6a displays the predicted $\mathrm{H}_{\alpha}$ equivalent width as a function of the star cluster mass, at the moment at which formation is completed and the cluster becomes visible for the first time $\left(W_{0}\left[\mathrm{H}_{\alpha}\right]\right)$. The continuous creation of stars during the formation phase leads to low-mass clusters with initial $\mathrm{H}_{\alpha}$ equivalent widths $\left(W_{0}\left[\mathrm{H}_{\alpha}\right]\right)$ larger than those arising from more massive clusters (see Fig. 6a).

After formation the number of UV photons drops rapidly and the $\mathrm{H}_{\alpha}$ equivalent width accordingly decreases (Fig. 6b). The evolutionary plot implies an HII region life-time which is slightly shorter than $10 \mathrm{Myr}$ after cluster formation, with an initially smaller $\mathrm{H}_{\alpha}$ equivalent width for larger masses of the resultant star clusters.
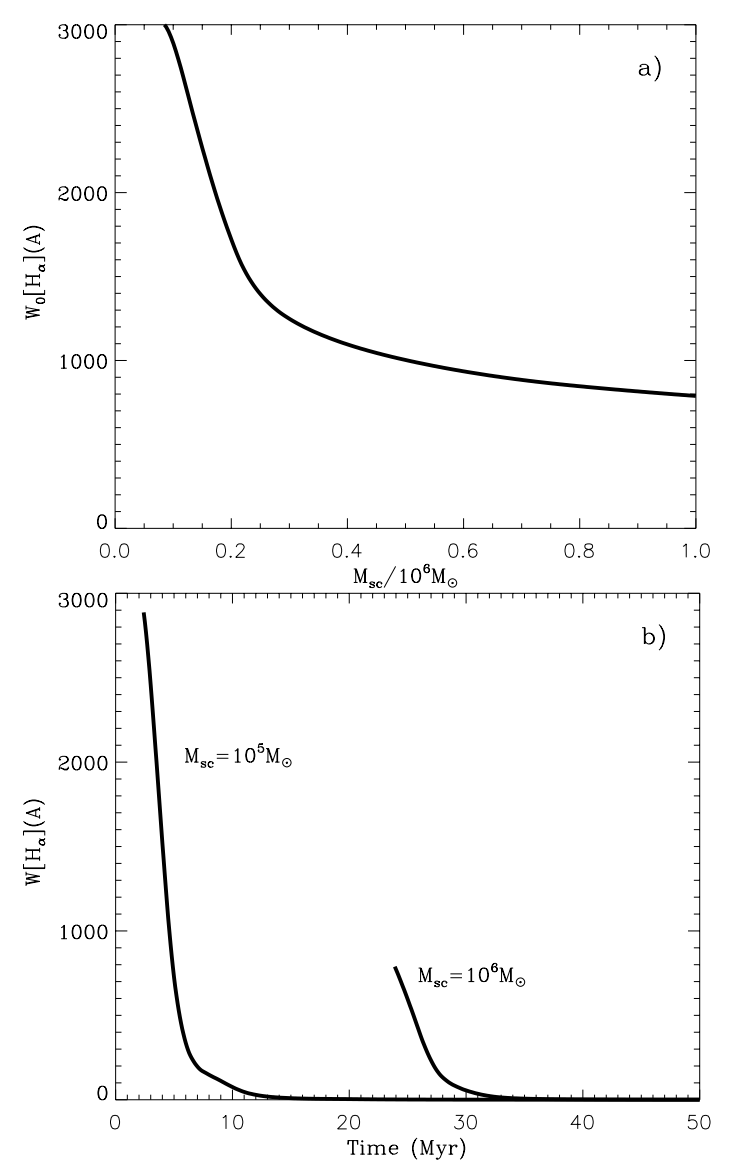

Fig. 6. The $\mathrm{H}_{\alpha}$ equivalent width. a) The $\mathrm{H}_{\alpha}$ equivalent width $W_{0}$ predicted at the moment when formation is completed and the clusters becomes visible, as function of the exciting star cluster mass. The synthesis models assumed the same upper and lower stellar mass limits, as well as the increasing star formation rate and the slope $(\alpha=-2.25)$ as in Fig. 1. b) The post-formation time evolution of the $\mathrm{H}_{\alpha}$ equivalent width for star clusters with a mass of $10^{5} M_{\odot}$ and $10^{6} M_{\odot}$.

\section{Conclusions}

The formation of compact and massive stellar clusters is naturally explained in the framework of the star-forming factory. The shell that stores the collapsing cloud is able to maintain its standing location and fragmenting properties, thanks to the larger energy input rate that results from the increasing number of massive stars in the central region. With this energy input rate, the forming cluster is able to balance the increasing gravitational pull exerted on the standing shell by the continuously growing mass in stars within the central region. Self-regulation in the factory can only be sustained for up to 20-25 Myr, while the mechanical energy input rate grows to satisfy the required energy to keep the shell in its standing location. Afterwards, the energy input rate grows and exceeds the equilibrium condition, causing the acceleration of the shell and with it its disruption as it moves into the skirt of the collapsing cloud. After that time, a few times $10^{6} M_{\odot}$ would have been converted into stars, all following a similar IMF. Smaller proto-cluster clouds are fully processed on shorter time-scales.

Small clusters $\left(\leq 2.7 \times 10^{5} M_{\odot}\right)$ would present a metallicity identical to the metallicity of the ISM out of which they 
formed. More massive clusters however, will carry the footprint of self contamination, produced by the supernovae products from former stellar generations used to support the factory at work. Massive clusters will then present a metallicity spread that would range from their host galaxy ISM metallicity at the moment of formation, to values similar to solar metallicity (see Fig. 5).

Upon formation, the more massive the resultant cluster, the smaller its initial $\mathrm{H}_{\alpha}$ equivalent width. This may differ by factors of three between clusters with a final stellar mass of $10^{5}$ and $10^{6} M_{\odot}$. Large IR luminosities $\left(\geq 10^{8} L_{\odot}\right.$ are predicted for factories leading to large $\left(\geq 10^{6} M_{\odot}\right)$ super-star clusters.

A definite prediction of the factory model, applicable in particular to high mass clusters (say $\sim 10^{6} M_{\odot}$ ), is the possibility of finding, after formation, a mixture of stellar populations with different ages and metallicities. For example, within a massive cluster the model predicts the co-existence of $\mathrm{O}$ stars and supergiants evolving at the same time as WR stars, while other stars may explode as SN. All of this is the result of the evolution of consecutive generations of stars born at different stages during the formation of the cluster.

Acknowledgements. We wish to express our thanks to Café Slavia and to the Astronomical Institute, Academy of Sciences of the Czech Republic (Prague). Also to the INAOE (Puebla, México) and the IAG (Sao Paulo, Brazil) for their hospitality and the atmosphere that favored our collaboration. Our special thanks to our referee for a report full of ideas and suggestions to improve our work. Our thanks to B. G. Elmegreen for many clarifying remarks and to Monica Rodriguez and Elena Terlevich for their suggestions regarding the metallicity of our clusters. We also thank D. Rosa for his advise regarding the starburst synthesis models and to P. Kroupa for his comment on the fraction of the gas that can be removed from the parental cloud. We thank Enrique Perez J., Steve N. Shore and Bill Wall for comments and suggestions. JP gratefully acknowledges financial support from the Grant Agency of the Academy of Sciences of the Czech Republic under grants No. A3003705, K1048102 and AVOZ 1003909, GMT is partially supported by the Brazilian agencies FAPESP and CNPq, GTT, SS and CMT acknowledge support from the Conacyt (Mexico) grant 36132-E and the Consejo Superior de Investigaciones Cientificas grant AYA2001 - 3939.

\section{References}

Ashman, K. M., \& Zepf, S. E. 2001, AJ, 122, 1888

Binney, J., \& Merrifield, M. 1998, Galactic Astronomy. (Princeton, New Jersey: Princeton University Press), Ch.5

Bodenheimer, P., \& Steigart, A. 1968, ApJ, 152, 515

Bonner, W. B. 1956, MNRAS, 116, 351

Brown, J. H., Burkert, A., \& Truran, J. W. 1991, ApJ, 376, 115

Brown, J. H., Burkert, A., \& Truran, J. W. 1995, ApJ, 440, 666

Colina, L., Gonzalez-Delgado, R., Mas-Hesse, M., \& Leitherer, C. 2002, ApJ, 579, 545

Comeron, F. 1997, A\&A, 326, 1195

Cox, D. P. 1983, ApJ, 265, L61 de Grijs, R., O’Connell, R. W., \& Gallagher, J. S. 2001, AJ, 121, 768

Ebert, R. 1955, Z. Astrophys., 36, 222

Elmegreen, B. G. 1994, ApJ, 427, 384

Elmegreen, B. G., \& Efremov, Yu. N. 1997, ApJ, 480, 235

Elmegreen, B. G., Efremov, Yu. N., \& Larsen, S. 2000, ApJ, 535, 748

Foster, P. N., \& Chevalier, R. A. 1993, ApJ., 416, 303

Franco, J., Plewa, T., \& Garcia-Segura, G. 1997, Rev. Mex. Astron. Astrofis., Conf. Ser., 6, 172

Gorjian, V., Turner, J. L., \& Beck, S. C. 2001, ApJ, 554, L29

Harris, W. E., \& Pudritz, R. E. 1994, ApJ, 429, 177

Ho, L. C. 1997, Rev. Mex. Astron. Astrofis., Conf. Ser., 6, 5

Holweger, H. 2001, in Solar and Galactic Composition, ed. R. F. Wimmer-Schweingruber (New York: Springer-Verlag), AIP Conf. Proc., 598, 23

Hoyle, F. 1953, ApJ, 118, 513

Hunter, D. A., Light, R. M., Holtzman, J. A., et al. 1997, ApJ, 478, 124

Kobulnicky, H. A., \& Johnson, K. E. 1999, ApJ, 527, 154

Johnson, K. E., Kobulnicky, H. A., Massy, P., \& Conti, P. S. 2001, ApJ, 559, 864

Kroupa, P. 2001, MNRAS, 322, 231

Kroupa, P. 2002, Science, 295, 82

Larsen, S. S. 1999, A\&AS, 139, 393

Larsen, S. S., \& Richtler, T. 2000, A\&A, 354, 836

Larson, R. B. 1969, MNRAS, 145, 271

Larson, R. B. 1987, in Starbursts and Galaxy Evolution, ed. T. X. Thuan, T. Montmerle, \& J. Tran Thanh Van (Gif Sur Yvette, France: Éditions Frontières), 467-482

Leitherer, C., \& Heckman, T. M. 1995, ApJS, 96, 9

Mac Low, M.-M., \& Klessen, R. S. 2003 [astro-ph/0301093]

Mas-Hesse, J. M., \& Kunth, D. 1991, A\&AS, 88, 399

Moffat, A. F. J. 1997, Rev. Mex. Astron. Astrofis., 6, 108

O'Connell, R. W., Gallagher, J. S., Hunter, D. A., \& Colley, W. N. 1995, ApJ, 446, L1

Osterbrock, D. E. 1989, Astrophysics of Gaseous Nebulae and Active Galactic Nuclei (Mill Valley, California: Univ. Sci. book), 408

Paresce, F., \& De Marchi, G. 2000, ApJ, 534, 870

Piotto, G., \& Zoccali, M. 1999, A\&A, 345, 485

Salpeter, E. E. 1955, ApJ, 121, 161

Scalo, J. M. 1986, Fund. Cosmic Phys., 11, 1

Silich, S., Tenorio-Tagle, G., Terlevich, R., Terlevich, E., \& Netzer, H. 2001, MNRAS, 324, 191

Silich, S. A., Tenorio-Tagle, G., Muñoz-Tuñon, C., \& Cairos, L. M. 2002, AJ, 123, 2438

Tenorio-Tagle, G., Silich, S., Kunth, D., Terlevich, E., \& Terlevich, R. 1999, MNRAS, 309, 332

Thielemann, F.-K., Nomoto, K., \& Shigeyama, T. 1992, in Elements and the Cosmos, ed. R. J. Terlevich, B. Pagel, R. Carswell, \& M. Edmunds (Cambridge University Press), 68

Vishniac, E. T. 1994, ApJ, 428, 186

Wünsch, R., \& Palouš, J. 2001, A\&A, 374, 746

Watson, A. M., Gallagher, J. S. III, Holtzman, J. A., et al. 1996, AJ, 112,534

Whitmore, B. C., Zhang, Q., Letherer, C., et al. 1999, AJ, 118, 1551

Wyse, R. F. G. 1997, ApJ, 490, L69

Zhang, Q., \& Fall, M. S. 1999, ApJ, 527, L81 